gewöhnlichen Ausführung der Schlachtung werthlos. Fleisch kann hierdurch nicht konservirt werden, weil die in den zahlreichen Falten, Nischen und Höhlungen der Fleischtheile haftenden Keime und namentlich die durch Messerstiche etc, in die tieferen Schichten gelangten Bakterien und Schimmelpilze durch das Aufstreuen von Konservirungsmitteln, wie Borsäure etc., oder durch das Einstäuben von desinficirenden Flüssigkeiten nicht getroffen und geschädigt werden. Solches Fleisch unterliegt trotz überreicher Anwendung ron Borsäure, Ameisensäure oder anderen Konservirungsmitteln, in kurzer Zeit dem Verderben.

Als ein Beispiel eines erfolgreichen, hauptsächlich auf reinliche Schlachtung sich gründenden Verfahrens der Fleischkonservirung beschreibt Emmerich die von Dr. Deichstätter und ihm ausgearbeitete Methode und macht dabei auf die Forderungen aufmerksam; welche bei der Aufbewahrung und Konservirung von Fleisch und Fleischwaaren überhaupt im Interesse der Gesundheit der Konsumenten erfüllt werden müssen. Das Verfahren besteht ausser der aseptischen Schlachtung im Besprühen der Schnittflächen bezw. Fleischoberflächen mit Eis= essig und Einpacken der geschlachteten ganzen Thiere oder der Fleischstücke in sterilisirte Sägespähne.

Prof. Emmerich verzichtet darauf bestimmte Resolutionen in Vorschlag $\mathrm{zu}$ bringen, glaubt aber, dass die freie Vereinigung bayerischer Vertreter der angewandten Chemie der Sache auch in Zukunft ihr Interesse zuwenden und späterhin bestimmte Regeln für die Ausführung der Schlachtung und die Behandlung von Fleisch und Fleischwaaren vereinbaren sollte, welche alsdann in den ortspolizeilichen Vorschriften, betreffend den Verkehr mit Nahrungsund Genussmitteln, sowie in den Schlachtvorschriften der Städte Aufnahme finden dürften.

\title{
Wann ist eine Fleischwaare als rerdorben zu betrachten?
}

\section{Von}

\author{
Dr. C. Mai in München.
}

Vortrag, gehalten auf der 19. Jahresversammlung der freien Vereinigung bayerischer Vertreter der angewandten Chemie zu Bamberg.

Wohl kaum eines der wichtigeren Nahrungsmittel ist - namentlich in den heisseren Jahreszeiten und bei mangelhafter Aufbewahrung - so leicht dem "Verderben" ausgesetzt, als das Fleisch und die verschiedenen Arten der Fleischwaaren. Häufig tritt daher an den Nahrungsmittelchemiker die Aufgabe heran, das "Verdorbensein" einer Fleischwaare auf Grund chemischer Untersuchung und unter Angabe zuverlässiger Thatsachen mit Sicherheit festzustellen.

Fs darf wohl ausgesprochen werden, dass seine Lage in diesem Falle keine ganz leichte und besonders angenehme ist. Denn die Methoden, die ihm hierbei zur Verfügung stehen sind bekanntermassen mehr als dürftig. So erklären 
auch z. B. die "Vereinbarungen zur einheitlichen Untersuchung und Beurtheilung von Nahrungs- und Genussmitteln etc. für das deutsche Reich" den Nachweis der Fleischfäulniss in erster Linie als Sache des Thierarztes und beschränken die chemische Untersuchung auf den etwaigen Nachweis der aromatischen Oxysäuren, des Indols und Skatols, sowie' der Phenole nach Baumann und Hoppe-Seyler.

Eine Klärung dieser keineswegs unwichtigen Frage erschien somit umso wünschenswerther, als auch die über diesen Gegenstand vorhandene Litteratur, soweit von einer solchen überhaupt gesprochen werden kann, sich sehr in Widersprüchen bewegt.

Herr Hofrath Hilger, auf dessen Veranlassung ich als Berichterstatter in dieser Frage heute auftrete, hat deshalb in dankenswerther Weise die Ausfiihrung einer grösseren Reihe von Versuchen veranlasst, die im Laboratorium für angewandte Chemie der Universität München unter Mitwirkung ron Herrn Dr. W. Bonhon angestellt wurden und deren Hauptzweck der war, festzustellen, ob und wie beginnende Fleischfäulniss chemisch nachweisbar ist.

Es wurden hierzu die verschiedenartigsten thierischen Stoffe, wie Rind- und Pferdefleisch, Leber und Gedärme von Rind und Pferd, ferner Blut, Lunge, Leber, Herz und Gedärme rom Schwein, sowie die verschiedenen frischen Wurstsorten und Leberkäse in den Untersuchungsbereich gezogen. Diese Objekte wurden dann unter Umständen, die denen möglichst nachgebildet waren, wie sie sich in der Praxis ergeben, der Fäulniss überlassen und versucht, in den verschiedenen Stadien der Zersetzung bestimmte chemische Individuen zu fassen, die für das betreffende Stadium charakteristisch sind.

Als solche Körper galten insbesondere Ammoniak, Amine, Oxysäuren, Sohwefelwasserstoff, Ptomaïne, Indol, Skatol u. s. w.

Es wärde hier zu weit führen und ist auch nicht meine Absicht, die Einzelheiten aller dieser Versuche anzuführen, da hierïber später noch eingehend berichtet werden soll; ich möchte vielmehr nur die Hauptergebnisse dieser Versuche kurz zusammenfassen.

Es zeigte sich zunächst, dass gerade das Stadium der beginnenden Fleischfäulniss, also dasjenige, das den Nahrungsmittelchemiker eigentlich am meisten interessirt, so gut wie gar keine bestimmten chemischen Merkmale aufweist, und dass solche erst bei fortschreitendem Zerfall auftreten, zu einer Zeit, wo der chemische Nachweis einer Fäulniss schon deshalb eigentlich unnöthig ist, weil sich Verdorbensein und ekelerregende Beschaffenheit des betreffenden Objektes dann schon in anderer Weise, nämlich in der äusseren Beschaffenheit, durch den Geruch u. s. w. zu erkennen geben.

Vor allen Dingen konnte festgestellt werden, dass Indol und Skatol, also gerade die von den "Deutschen Vereinbarungen“ zur Erkennung der Fäulniss hervorgehobenen Körper unter keinen Umständen als Erkennungsmittel beginnender Fleischfäulniss in Betracht kommen können. Bei keinem der Versuche war es möglich, diese Körper auch nur spurenweise nachzuweisen; ihr Nach- 
weis gelang rielmehr nur in Massen, die bereits in hochgradigem Zerfall begriften waren und bei denen die Nothwendigkeit eines chemischen Nachweises der Fäulniss jedenfalls nicht mehr vorlag.

Ebensowenig ist der Nachweis von Ptomaïnen geeignet, beginnende Fäulniss zu beweisen, da auch sie erst im Stadium fortschreitender Zersetzung auftreten, ganz abgesehen davon, dass ihre Isolirung und bestimmte Charakterisirung ausserordentlich umständlich und überhaupt nur mit grossen Materialmengen möglich ist.

Was nun den quantitativen Nachweis ron Ammoniak betriffit, so kann dieser für sich allein ebenfalls nicht als Maassstab der Fäulniss betrachtet werden, da sowohl die normalen, wie die bei der Zersetzung entstehenden Ammoniakmengen ziemlichen Schwankungen unterworfen sind; letztere nehmen bei beginnender Fäulniss allerdings zunächst zu, vermindern sich aber wieder, um später wiederum anzusteigen. Bessere Anbaltspunkte giebt dagegen das Verhältniss des Ammoniaks zum Gesammtstickstoft, welche beiden Bestandtheile in ein und derselben Fleischwaare in gewissen Beziehungen zu einander zu stehen scheinen. In frischer Rindsleber z. B. beträgt der Gesammtstickstoff $3,1 \%$, der Ammoniakgehalt $0,32 \%$; letzterer steigt nach 4 Tagen schon auf $0,42 \%$. Im frischen Gedärme des Schweines stellt sich der Stickstoffgehalt auf 2,2\%, das Ammoniak auf $0,22 \%$; nach 3 Tagen schon auf $0,35 \%$. Leberkäse enthielt $2 \%$ Gesammtstickstoff und $0,23 \%$ Ammoniak, das nach 3 Tagen auf $0,32 \%$ gestiegen war.

Wir sehen also, dass die Ammoniakmenge, die in den frischen Waaren etwa 10\% des Gesammtstickstofies ausmacht, nach 3-4 Tagen schon eine beträchtliche Steigerung erfährt.

Besonders bemerkenswerth ist aber das Verhalten der Umhüllungsmembranen des Verdauungsapparates, der Gedärme u. s. w. Diese lassen schon imı ersten Beginn einer Zersetzung dentlich die Entstehung von Schwefelwasserstoff, wenn auch nur in lieinen Mengen erkennen, zu einer Zeit, in der z. B. bei Würsten der von dem betreffenden Darm eingeschlossene Inhalt noch völlig unverdorben ist. Jedenfalls ist dieses Verhalten geeignet, beginnende Zersetzung bei Wurstwaaren erkemen zu lassen.

Aminbasen treten ebenfalls schon bei beginnender Fleischfäulniss auf, jedoch nur in so geringen Mengen, dass sie lediglich durch den Geruch in den Destillaten erkennbar sind. Mit fortschreitender Zersetzung werden die Mengen dann grösser und auch chemisch nachweisbar. Auch hierin zeigen die Darmmassen wieder ein rom Muskelfleisch abweichendes Verhalten, indem bei ihnen schon nach 16 Tagen grössere Mengen Trimethylamin erkennbar sind, während dies z. B. bei Leber etc. nach dieser Zeit kaum spurenweise vorhanden ist.

Das Auftreten grösserer Mengen ron Aminbasen, sowie der flüchtigen Fettsäuren, Butter-, Valerian- und Capronsäure, ferner der Amidosäuren, Glykocoll, Leucin und Tyrosin geht schon mit einer tiefergreifenden Zersetzung Hand in Hand, doch machen auch hier wieder die Darmmassen insofern eine Ausnahme, 
als in diesen die genannten Körper viel früher und in grösseren Mengen entstehen, als im Muskelfleisch.

Fassen wir also die beobachteten Erscheinungen etwas näher ins Auge, wie sie sich beim Lagern von Fleischwaaren, Würsten u. s. w., die sich als Gemenge von Proteïnsubstanzen, Fett, Blut und thierischen Membranen darstellen, unter normalen klimatischen Verhältnissen ergeben, so können wir folgende Stadien fortschreitender Zersetzung mit Hilfe der dabei auftretenden Körper unterscheiden.

Im ersten Stadium lassen sich chemisch charakterisirbare Körper als Zersetzungsprodukte nicht nachweisen, doch beginnt alsbald, nach 3-4 Tagen schon das Verhältniss des Ammoniaks zum Gesammtstickstoff sich merklich zu verschieben.

Das zweite Stadium beginnt mit dem Auftreten nachweisbarer Mengen von Aminbasen der aliphatischen Reihe, insbesondere von Trimethylamin. Auch lassen sich in diesem Stadium die Amidosäuren leicht nachweisen.

Im dritten Stadium, dem Zustande fortschreitenden Zerfalles, der sich natürlich schon äusserlich durch den Geruch u. s. w. erkennen lässt, rerschwinden die Amidosäuren wieder und an ihre Stelle treten die Fettsäuren, sowie auch zuweilen Indol und Skatol. Auch die Amine haben sich jetzt so angereichert, dass ihre Isolirung mit Leichtigkeit gelingt. Endlich ist das Auftreten von Ptomaïnen, z. B. von Putrescin erkennbar.

Im vierten Stadium endlich verschwinden die genannten Körper allmählich wieder, indem mit fortschreitendem Zerfall als basische Zersetzungsprodukte immer einfachere Körper entstehen, bis schliesslich nur noch Ammoniak vorhanden ist.

Für den Nahrungsmittelchemiker kommen natürlich nur die beiden ersten Stadien in Betracht; erhebt sich der Ammoniakgehalt einer Fleischwaare iiber die normale Grenze, die allerdings noch näher zu präzisiren sein wird, und wird das Auftreten von Trimethylamin in mehr als spurenweiser Menge erkennbar, so wird die Waare als verdorben rom chemischen Standpunkte aus zu bezeichnen sein. Fine abweichende Beurtheilung erfordern die zum Umhüllen der Wurstmassen dienenden Därme, bei denen das frühzeitige Auftreten von Schwefelwasserstoff, sowie verhältnissmässig grösserer Mengen von Aminen und Fettsäuren, sowie auch ron Indol und Skatol kennzeichnend ist. Bei der Beurtheilung von Wurstwaaren wird man daher hauptsächlich das Augenmerk auf den umbüllenden Darm und die zunächst darunter liegenden Theile des Inhaltes zu richten haben, da diese Theile der Zersetzung am frühesten anheimfallen. 